\title{
LEVERAGING AGENT BASED SIMULATION FOR RAPID COURSE OF ACTION DEVELOPMENT
}

\author{
Philip S. Barry \\ Matthew T. K. Koehler \\ Mailstop: H305, W903 \\ 7515 Colshire Dr. \\ The MITRE Corporation \\ McLean, VA 22102 U.S.A.
}

\begin{abstract}
In the spring of 2005 a limited objective experiment was carried out to assess the feasibility of using agent based simulations to enhance co-evolutionary course of action development. In particular, relatively low fidelity simulations were employed to visualize the results of particular courses of action. Over four days multiple courses of action were developed by two opposing teams with similar force structures and then run against one another in an agent based modeling environment to test their ability to achieve the given mission. The results of the experiment indicate that there is significant potential for low fidelity simulations to stimulate objective thinking in course of action development.
\end{abstract}

\section{INTRODUCTION}

A limited objective experiment was conducted under the purview of the Marine Corps' Project Albert to investigate and assess the utility of using agent based models and data farming/data mining techniques to assist Course of Action (COA) Development. The experiment brought together an international team of military and technical experts that used agent based modeling and data farming to help develop and assess a series of COAs. The product of this group was the validation of a concept of simulation assisted COA development and lessons learned on how to integrate these techniques into a military planning cell.

This paper begins with a brief background of Project Albert and an introduction to the concept of data framing. From there, a discussion of a methodology for using low resolution simulations and data farming to aid in the development of COAs is be presented. The remainder of the paper describes the limited objective experiment run in the spring of 2005. The paper concludes by examining the implications of the experiment.

\section{BACKGROUND}

Project Albert (Brandstein and Horne 1998, Fry and Forsyth 2002) has been driven by the idea that low fidelity models run millions of times can provide insights into nonlinearities, intangibles, adaptation and leverage points that might otherwise remain undiscovered using traditional modeling techniques. Project Albert is comprised of two major technical components; data farming and the low fidelity agent based models that are used to examine problems of interest. This paper will focus primarily on the use of Data Farming in the context of developing courses of action. For a more detailed examination of the agent based modeling environments and applications of the modeling environments, the reader is directed to Barry and Koehler (2004), Lauren (2002), and Lauren (2000).

\subsection{Data Farming}

Data Farming is a broad term that encompasses many separate processes that form a cohesive whole but all begin with a question of interest. The question is usually formulated by an analyst, subject matter expert, or decision-maker who collaborates with a modeler to create - in a matter of days or hours - a model to explore the essence of the question at hand. There are a variety of modeling environments that facilitate the generation of the agent based models. The maturity of these environments makes rapid model generation feasible, providing the opportunity to develop several models for a given question. The use of these low fidelity models also increases the importance of the subject matter expert because they must be able to distill the situation, as defined by the question, to its most relevant features.

There are three aspects to how the model is run within the data farming environment. First, a large parameter space is explored in either a full factorial experimental design or some other sampling regime, such as a NearOrthogonal Latin Hypercube (Lucas et al. 2002). Second, 
due to the sensitivity of the models to slight perturbations in the initial layout or the random number stream during the run, the simulations runs will often use the same parameter combinations with different random seeds. Finally, the parameter combinations may not be set initially, but may be created using one of a number of different evolutionary or natural algorithms to find nearoptimal parameter combinations based upon a user defined fitness function.

When the model runs are completed the output data is analyzed to determine if the model was created correctly, and if it adequately captured the essence of the question. If problems arise with either how the model was created or how it captured the question, the model is quickly modified and run again. When the modeler and subject matter expert are satisfied that the model represents the question at hand, the analysis enters the Operational Synthesis (Horne 2001) cycle where the modeler and the subject matter expert seek to develop insights into the problem at hand. The term insight here is not used gently-these models are abstractions and not designed to provide detailed engineering level solutions. However, it has been shown that the models such as these can be used to inform other aspects of the analytic processes, be they legacy models, traditional decision support, or even a war game as discussed in Cioppa et al. (2004).

\subsection{Data Farming Enhanced Course of Action Development}

COAs are currently developed in a planning cell and then evaluated via visual inspection by subject matter experts (SMEs). In general, three COAs for a given operation are developed and then presented to the SMEs who evaluate them from the perspective of how the opposing force may react. The "best" COA of the three developed is selected. However, there is usually not enough time to thoroughly test each COA, particularly for all of the many ways that the opposing force (OPFOR) may react. This may produce COAs that have undiscovered weaknesses and vulnerabilities. Herein lies the tension: how does one adequately test a larger set of COAs in a timeframe that is operationally viable?

To address this tension we advocate the use of low resolution, stochastic agent-based models. These models can be produced quickly and can be run in a supercomputing environment to allow for a large number of simulation executions that can be completed in a short period of time. While simulation has been used for COA development and analysis in the past, much of the previous work has tended to remove the human from the COA development loop. Without human participation, the simulation setup was generally too complex for rapid setup and large parameter exploration. The approach described here creates a methodology in which humans create the $\mathrm{COA}$ and then use simulation to visualize and test the COA thoroughly and quickly. Once this test is completed the COA may be changed or thrown-out all together, thus initiating another round of simulation testing. This allows for a co-evolutionary cycle between the planners and the OPFOR staff. This evolutionary cycle was one of the foci of the experiment. COAs were developed by a Blue force and an OPFOR or Red force. The developed COAs were then run against each other to evaluate their fitness. Then each side was given the opportunity to adjust their COAs. The cycle then continued with the new COAs. As each COA has a large number of parameters, the number of possible parameter combinations and potential outcomes that may happen is far too large for a manual exploration. Therefore, we employ data farming to investigate how each COA will perform.

\section{THE LIMITED OBJECTIVE EXPERIMENT}

During one week in May of 2005 the limited objective experiment was conducted to investigate and assess the utility of using the aforementioned agent based models and data farming/data mining techniques to assist in Course of Action development. A team of military and technical experts from several countries were brought together to use Project Albert models and data farming to plan an operation.

After introductory briefings, the group was divided into two sub-teams who independently conducted planning for a notional Blue force and a Red force. Both teams made use of Project Albert tools to both design and evaluate their respective COA. At the end of each day the teams submitted COAs that, while developed independently, were evaluated against one another by running them in the data farming environment.

\subsection{The Operational Scenario}

Both forces started out several kilometers from an airfield in different parts of the area of operation (AO). The scenario takes place at the intersection of three nations, $\mathrm{A}$, B, and C. Nations A and B (upper left and upper right of the Figure 1, respectively) are on the brink of outright war. They both need an airfield, however. The closest useable airfield is in country $\mathrm{C}$ (the lower portion of Figure 1, the airfield is outlined by the triangle). The mission of both the Red and Blue forces is to take control of the airfield, deny access of it to the other side, and hold the airfield until reinforcements arrive.

A detailed set of forces and associated behaviors was modeled using MANA. Digital elevation data from Camp Pendleton was fully integrated into the scenario files as 


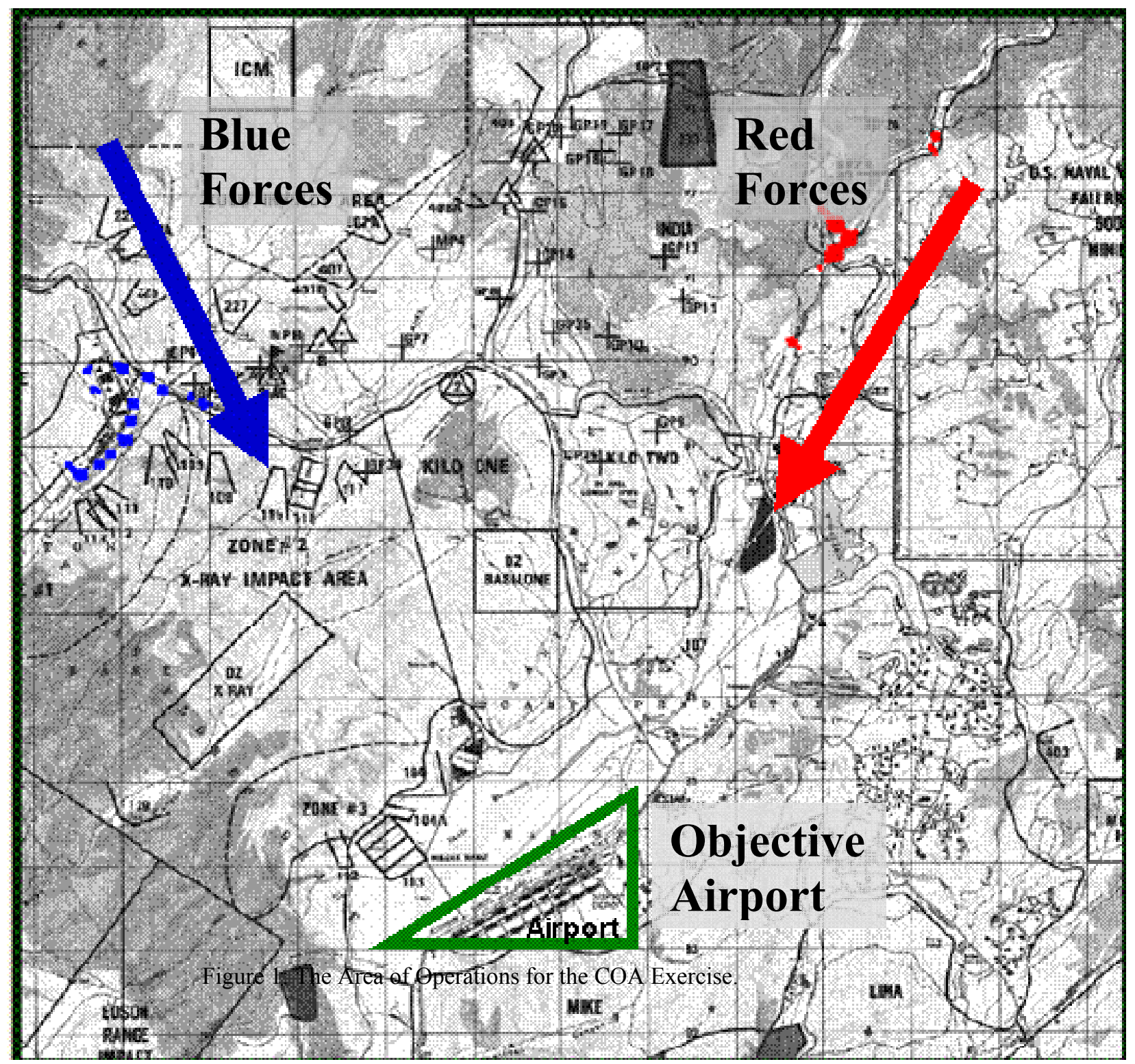

Figure 1: The Area of Operations for the COA Exercise.

well as the appropriate map displays. Furthermore, GIS data was used to create the movement constraints for the agents. Paper maps were provided for planning purposes. A clear definition of parameters that were to be farmed (e.g., propensity to engage the opposing force) as well as those parameters that will be held fixed (e.g., probability of kill associated with each weapon) was discussed with the participants. Team members were sent the force structure, the area of operation, and a demo model prior to the workshop to encourage pre-experiment thinking as to specific tactics. Finally, the sub-teams were told ahead of time that their mission is to secure and airfield and repel any opposing actions. Both forces had similar military capability that included infantry, mechanized units and indirect fire weapons as howitzers as shown in Table 1.

\subsection{Terrain}

Terrain considerations are an essential part of the COA development process. Terrain affects movement speed (including becoming impassable), cover, concealment, and line of sight. In fact, during the experiment one Red COA rallied their forces in an area that was isolated from the airport (on the other side of a ridge) and thus, "gave" the airport to Blue because Red could not see, and therefore could not engage, Blue. 
Table 1: The Force Structure

\begin{tabular}{|l|l|}
\hline Entity & Quantity \\
\hline Task Force Commander & 1 \\
\hline Company Commander & 3 \\
\hline Rifle Platoons & 3 \\
\hline Weapons Platoon & 1 \\
\hline Machine Guns & 6 \\
\hline 60 mm Mortars & 3 \\
\hline LAWs & 3 \\
\hline Tanks & 4 \\
\hline LAVs & 16 \\
\hline EFVs & 15 \\
\hline TOWs & 8 \\
\hline Heavy Machine Guns & 6 \\
\hline Javelins & 6 \\
\hline $81 m m$ Mortars & 6 \\
\hline 155 Howitzer & 1 \\
\hline
\end{tabular}

Figure 2 depicts the terrain "as seen be agents" in the simulation. The dark color is passable off-road terrain. The yellow is passable roads. Light red is passable steep terrain, and dark red is too steep to move across. Light blue is water that is shallow enough to drive or walk through, whereas dark blue is too deep to drive or walk through.

\subsection{Data Farming}

Table 2 provides an overview of the data farming that took place during the COA experiment. A total of thirteen COAs were created during the experiment. The COAs were developed iteratively in order to allow for coevolutionary changes to take place between the two teams. Few external factors were varied to ensure that interpretation of the results was clear. Furthermore, the varied factors were limited to items that were deemed to be out of the control of either force, specifically communications accuracy, visibility and mobility as affected by weather and stealth (i.e., cover and concealment).

\subsection{Summary Data Farming Results}

As discussed above, the COAs were run against one another to determine the effectivity of the specific strategies developed. A straightforward measure of effectiveness for the COAs was chosen: forces have to capture the airport (see Figure 1) and control it until the end of the simulation run. Controlling the airport entailed having a force ratio greater than or equal to $3: 1$ within the airports boundaries. Further, the winning side had to have a least $30 \%$ of its forces remaining. By keeping the metrics for success simple less time was spent in data postprocessing which allowed for greater time in COA development.

Figures 3 and 4 highlight results from the final two COAs submitted by the Red and the Blue teams. As can be seen in Figure 3, Red only won when they used COA 1 and Blue used COA 2. Further, Red only won when it had a favorable loss exchange ratio. This pattern held for all of the simulation runs except for one, which is shown as the "outlier" in Figure 3. The outlier results show Red winning with an unfavorable loss exchange ratio. This is precisely the type of result that demonstrates the utility of data farming. In only 1 case of 150 runs of the simulation did Red win with an unfavorable loss exchange ratio. This type of result can be readily brought to the attention of analysts which can seek to understand under what conditions this might be relevant.

Figure 4 shows that Blue always wins against Red COA 2, whether or not Blue uses COA 1 or COA 2. However, the results show an interesting variability in the loss exchange ratio. For one group the loss exchange ratio is in Blue's favor. However for the other two groups the loss exchange ratio is in Red's favor even though the results show that Blue had won. Again, this visibility of this result is a direct result of the fact that data farming more fully explores the possible outcomes of the COA.

Table 2: Data Farming Overview

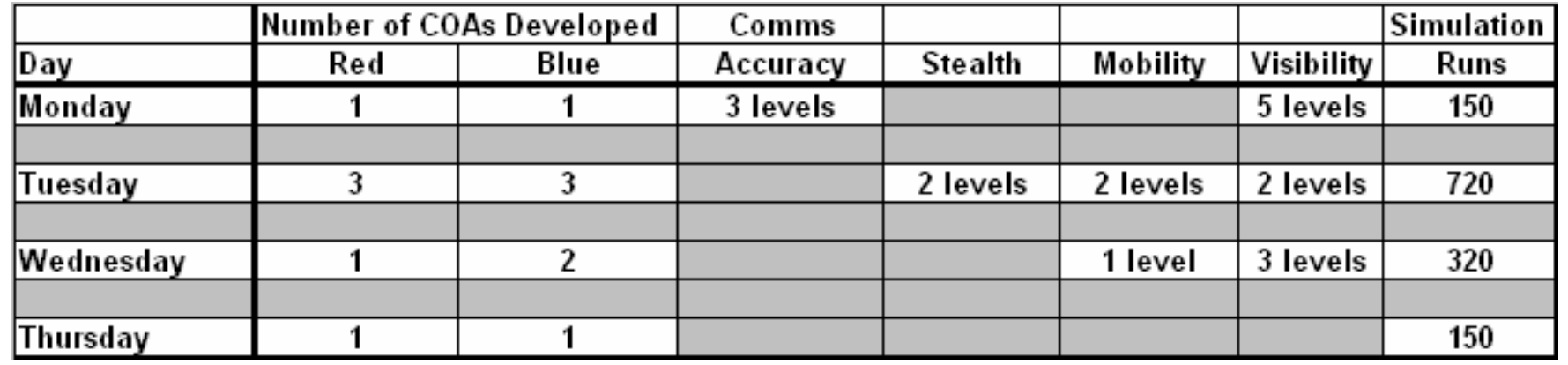




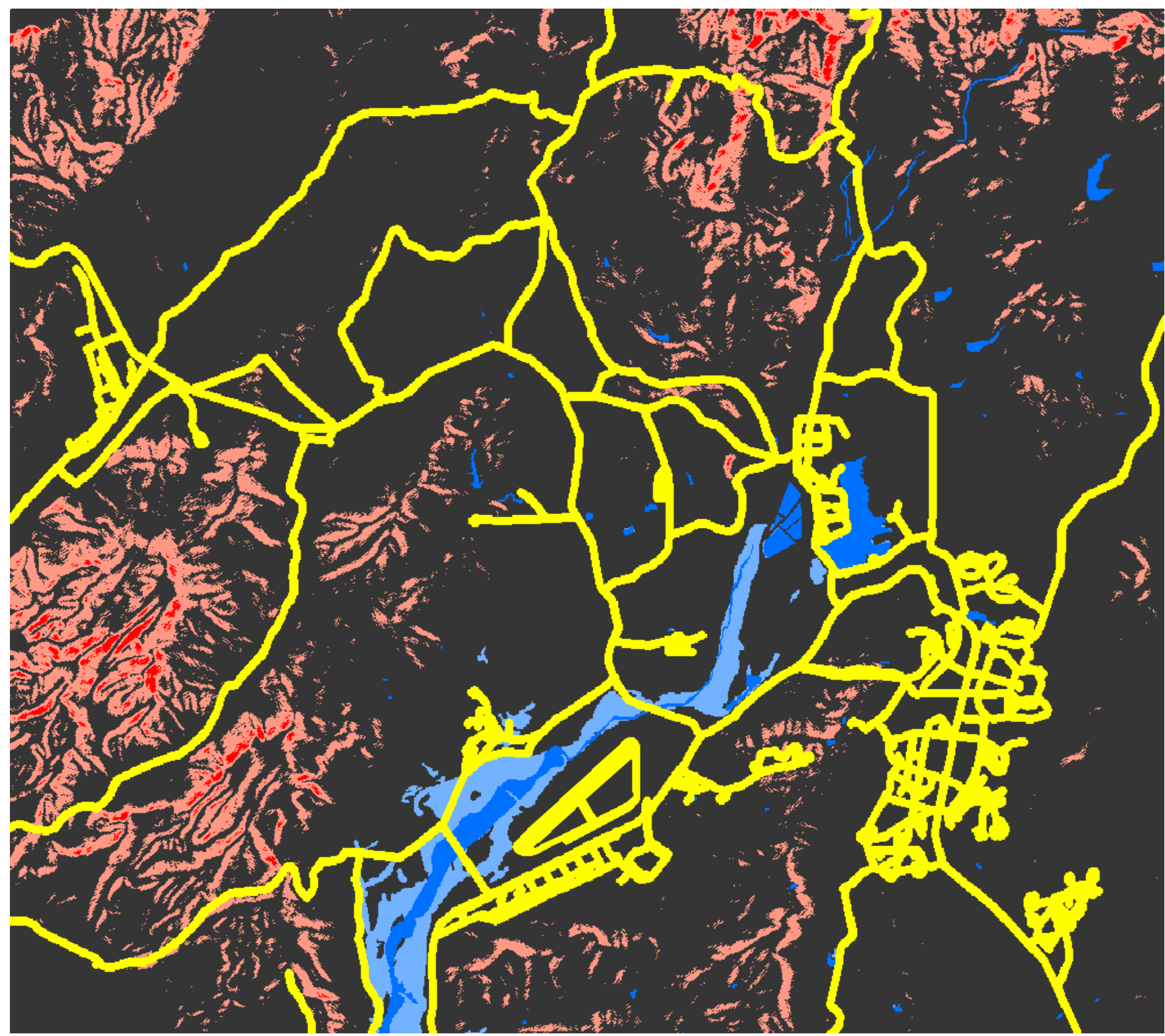

Figure 2: Terrain as Seen by Agents.

Should this have been a real exercise, further analysis would look into the difference between the three groups and select the COA where the loss exchange ratio was in Blue's favor.

\subsection{Assessment}

The military officers that were part of the concept demonstration indicated that the model has significant potential to support planning, particularly during the course of action development and COA wargame steps of the Planning Process. A clear benefit of using simulation is that it allows the planner to see or test the synchronization of the various elements of the COA. Specifically, simulation assisted COA development can investigate if the actions that make up the COA are arranged properly in time and space, with the express purpose of maximizing combat power at a decisive point. Watching the COA play out (as opposed to manually moving pieces in a traditional wargame) provided the planner in the experiment an opportunity to see how their concept of operations arranges the entire COA's actions from start to finish.

Unique to this approach is the empirical data it can provide the planner. As demonstrated in the experiment, planners can see the effects of weather, terrain, observation/visibility and enemy actions on their COA's as they relate to specific criteria such as speed, force protection, and command and control. Understanding how 


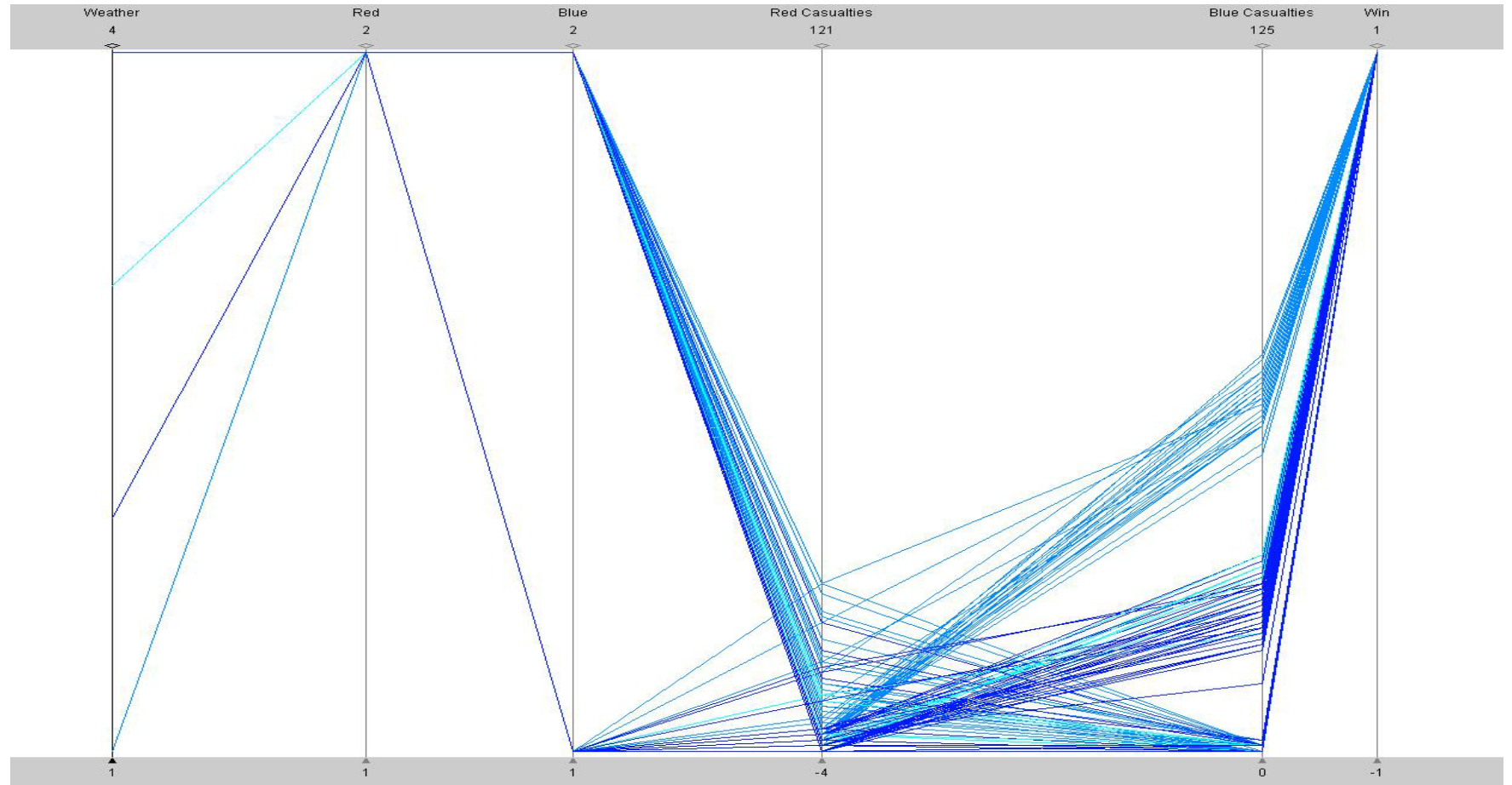

Figure 3: Red Only Wins with COA 2

factors effect the COA has the potential to help the planners understand the advantages and risks of the strategy chosen.

\section{SUMMARY}

The experiment demonstrated that the concept of using agent based models for rapid COA development is possible. In all, twelve COAs were developed in 48 hours. The process of simulating and data farming the COAs allowed the teams to gain a comprehensive overview of the planned operation and its phasing requirements. The simulation stimulated a learning environment that assisted in evolutionary COA development. While more work is

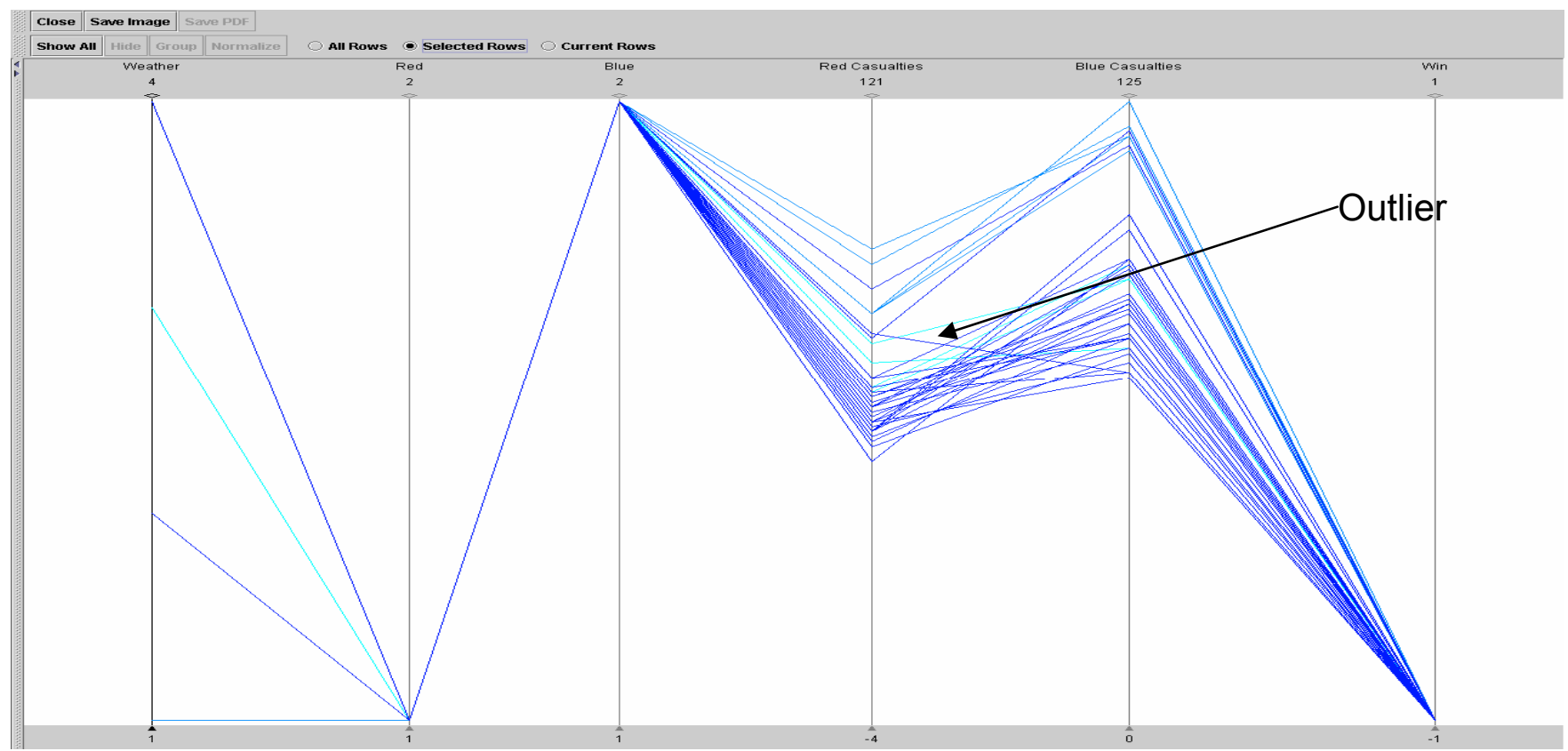

Figure 4: Blue Always Wins Against Red COA 1 
necessary to develop in depth analysis tools for analysis of the results from the simulations, insightful results were readily developed from the data farming provided during the week. It was agreed that with additional work this concept could be operationalized and introduced to a planning staff.

The experiment also demonstrated the use of data farming over a variety of COAs. Data farming enabled clear understanding of the attributes of force combinations within each scenario. This allowed for internal restructuring during the experiment, but has the potential to identify additional capabilities such as fire support that would have to be requested from other assets. As shown during the experiment, the planning staff can also test different mixes of capabilities, routes of movement and task organizations to reduce risk to the force.

Data farming also provided an opportunity for a more detailed analysis of the interactions of Blue and Red forces and provided an understanding of critical locations where preplanned fires and maneuver appeared to be more important. Interactions between Red and Blue forces within the model allowed the staff to achieve a more objective view of each COA and refined the ability of each member of the team to achieve a better understanding of the selected COA.

The military officers who participated in the experiment suggested two potential applications of this approach. One would use the agent based models and data farming to provide decision support to a staff in determining the time, space, terrain, and synchronization issues, as well as potential vulnerabilities of various tactics. The other would evolve a set of friendly COAs against possible enemy COAs and capabilities. Data farming would be used to help improve friendly courses of action. This would be an iterative process, with the modelers and planners working as a combined team to develop the best possible insights into the implications of the COAs.

\section{REFERENCES}

Barry, P. and M. Koehler. 2004. Simulation in context: using data farming for decision support. In Proceedings of the 2004 Winter Simulation Conference, ed. R. G. Ingalls, M. D. Rossetti, J. S. Smith, and B. A. Peters, 814 - 819. Piscataway, New Jersey: Institute of Electrical and Electronics Engineers. Available via http://www. informs sim.org/ wsc04papers/101.pdf [accessed July 15, 2005]

Brandstein, A., and G. Horne. 1998. Data farming: a metatechnique for research in the $21^{\text {st }}$ century. Maneuver Warfare Science 1998, ed. F. G. Hoffman and G. Horne. Washington, DC: US Marine Corps.

Cioppa, T. and T. Lucas, S. Sanchez, 2004. Military applications of agent based simulations. In
Proceedings of the 2004 Winter Simulation Conference, ed. R. G. Ingalls, M. D. Possetti, J. S. Smith, and B. A. Peters, 171-181. Piscataway, New Jersey: Institute of Electrical and Electronics Engineers. Available via http://www. informssim.org/wsc04papers/020.pdf [accessed July 15,2005$]$.

Fry, A., and A. Forsyth. 2002. The Australian army and Project Albert: pursuing the leading edge of military thinking and technological development. Maneuver Warfare Science 2002, ed. G. Horne and S. Johnson. Quantico, VA: US Marine Corps.

Lauren, M. 2002. A fractal based approach to equations of attrition. Military Operations Research Journal 7:3.

Lauren, M. 2000. Modeling combat using fractals and the statistics of scaling systems. Military Operations Research Journal 5:3.

Lucas, T., and S. M. Sanchez, L. Brown, W. Vinyard. 2002. Better designs for high-dimensional explorations of distillations. Maneuver Warfare Science 2002, ed. G. Horne and S. Johnson.

\section{ACKNOWLEDGMENTS}

The authors gratefully acknowledge the major contributions of Theodore Meyer, Steven Upton, Col Darryl Stanley, USMC (Ret), Cpt. Bain, USMC, and the participants of the experiment. Without these individuals the work discussed in this paper would not have been possible.

\section{AUTHOR BIOGRAPHIES}

PHILIP BARRY is a Senior Principal Engineer at The MITRE Corporation. He has been working in the modeling and simulation arena for a number of years, most recently with Project Albert. He has graduate degrees in Information Technology and Systems Engineering from George Mason University and an undergraduate degree in Aerospace Engineering from the University of Virginia. His email address is pbarry@mitre.org.

MATTHEW KOEHLER is an Artificial Intelligence Engineer at The MITRE Corporation. $\mathrm{He}$ is currently applying agent based modeling tools and techniques to the work of a number of government agencies. He has graduate degrees from the George Washington University School of Law and the School of Public and Environmental Affairs at Indiana University, and an undergraduate degree in Anthropology from Kenyon College. His email address is mkoehler@mitre.org. 